\title{
Reward value of attractiveness and gaze
}

\author{
Making eye contact enhances the appeal of a pleasing face, irrespective of gender.
}

$\mathrm{F}$ aces are visual objects in our environment that provide strong social cues ${ }^{1}$, with the eyes assuming particular importance ${ }^{2,3}$. Here we show that the perceived attractiveness of an unfamiliar face increases brain activity in the ventral striatum of the viewer when meeting the person's eye, and decreases activity when eye gaze is directed away. Depending on the direction of gaze, attractiveness can thus activate dopaminergic regions that are strongly linked to reward prediction ${ }^{4}$, indicating that central reward systems may be engaged during the initiation of social interactions.

In an event-related functional magnetic resonance imaging (fMRI) study, 16 subjects ( 8 male, 8 female) were shown colour images of 40 different faces, which had their eyes directed either at or away from the subject (Fig. 1). After the fMRI scanning session, subjects rated the attractiveness of the faces they had seen, and these ratings were normalized and parametrically entered into the

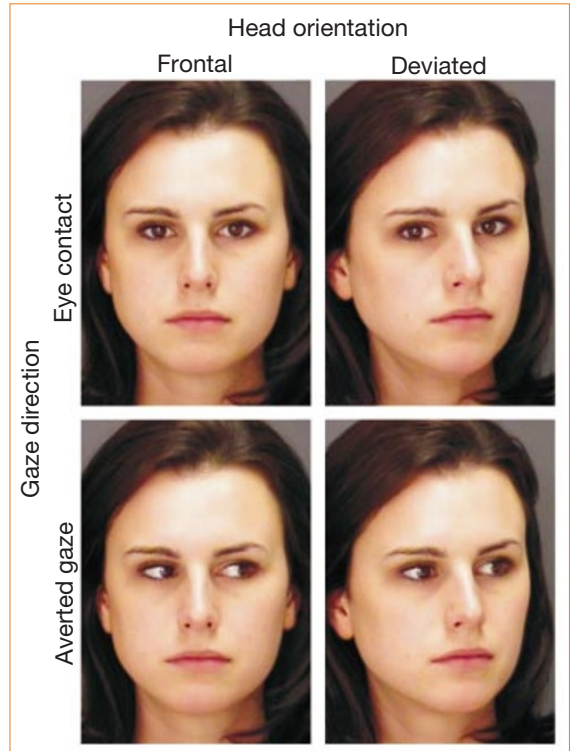

Figure 1 Examples of eye-contact and non-eye-contact stimuli. Faces were counterbalanced for head position, sex and, for non-eye-contact images, gaze direction (right or left). During each scanning session, subjects were presented with 2 runs of 80 faces and 40 scrambled control faces in a randomized, eventrelated design (new image every $3.5 \mathrm{~s}$; presentation time, $1.2 \mathrm{~s}$, with intervening scrambled images). No task was required from subjects while they viewed these images, but to secure and test their attention, six target images (faces with closed eyes) were included, to which subjects were asked to respond by pressing a button. In statistical analysis, these latter images were modelled as events of no interest and excluded from further evaluation. After scanning, subjects rated the attractiveness of the 40 faces on a scale from 1 to 10. There were no significant differences between ratings by male and female subjects, or between ratings on male and female faces. analysis. Significant effects $(P<0.05$, corrected for multiple comparisons) were assessed using a $t$-test and displayed as statistical parametric maps using SPM99b software.

No brain region showed any activation in response to facial attractiveness per se. We therefore investigated whether processing of attractiveness depends on gaze direction. When eye gaze was directed at the subjects, activity in the ventral striatum correlated positively with attractiveness (slope: $+0.46 \%$ change in signal intensity per standard deviation increase in attractiveness rating). In contrast, this correlation was reversed when eye gaze was averted, causing activity in the ventral striatum to decrease with increasing attractiveness (slope: $-0.88 \%$ signal intensity per s.d. increase in attractiveness).

Figure 2 shows the resulting activation map when these two contrasts are combined as an interaction - for example, the contrast (correlation with attractiveness when eye contact is made) minus (correlation with attractiveness when no eye contact is made). In a fixed-effects analysis, the effect was significant at a voxel level (the smallest volume tested in our study) in the right ventral striatum $(P=0.015)$ and at cluster level bilaterally (right, $P<0.01$; left, $P=0.01)$. We also implemented a secondlevel random-effects analysis to determine whether the results could be extended to the population at large. Significant effects persisted at a cluster level in the right ventral striatum $(P<0.01$; point of maximal activation: $x=12, y=-8, z=10)$ and approached significance in the left ventral striatum $(P=0.06, x=-10, y=0, z=4)$.

The gender of the observed face in relation to the gender of the observer did not influence the response in the ventral striatum. This might indicate that features depicting attractiveness (such as facial form and symmetry) are low-level cues that are processed independently of gender.

Our results provide evidence not only that the ventral striatum processes reinforcing stimuli that serve basic survival needs ${ }^{5}$, but also that it is more generally involved in the evaluation of stimuli with relevance to social interactions. The activity of dopaminergic neurons that project to the ventral striatum is associated with reward prediction $^{6}$. The firing rate of these neurons follows a specific pattern, increasing when an unexpected reward is seen and decreasing when an expected reward fails to materialize, thereby predicting error in future rewards.

In accordance with this specific pattern, we found no activation associated with

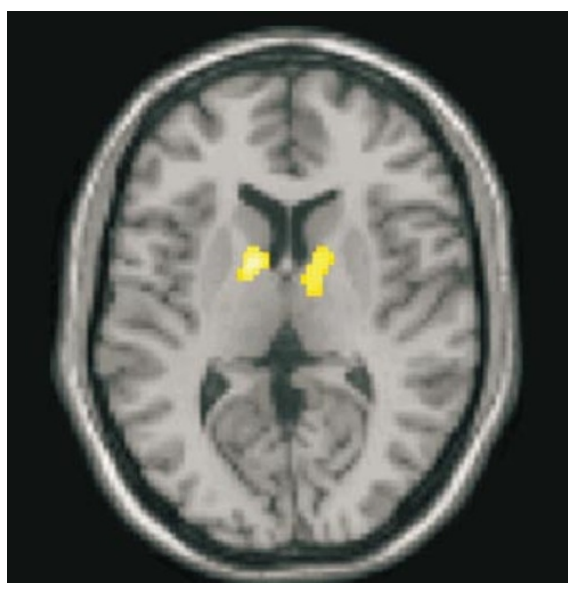

Figure 2 Depending on eye gaze, the degree of attractiveness of faces modulates activity in the ventral striatum, a brain area associated with reward prediction. When eye gaze is directed at a subject, the degree of attractiveness of individual faces correlates positively with brain activity in the ventral striatum; when eye gaze is directed away, this correlation is reversed and activity in the ventral striatum decreases with increasing attractiveness. Activation maps are superimposed on the surface of a T1-weighted template (fixed-effects analysis showing areas in which activity surpasses the threshold of $P=0.05$ at the cluster level. Time to repetition, $3.16 \mathrm{~s}$; 32 transverse slices; voxel size, $3 \times 3 \times 3 \mathrm{~mm}$; Siemens VISION 2T).

attractiveness per se, but only as an interaction with eye gaze. Assuming that the dimension of attractiveness constitutes a reward, especially when social interaction is initiated, returned eye gaze from an attractive face represents a more favourable result than expected, leading to enhanced responses, whereas failing to make eye contact with an attractive face is a disappointing outcome, leading to reduced activity in the dopaminergic target systems. Likewise, eye gaze from an unattractive face may result in disappointment and a reduced response, whereas missing eye contact with an unattractive face may be a relief and thus enhance activity. The profile of the response we describe here may reflect an automatic evaluation of the likely reward that can be derived from conspecifics.

Knut K. W. Kampe*, Chris D. Frith $\dagger$,

Raymond J. Dolan $†$, Uta Frith*

${ }^{*}$ Institute of Cognitive Neuroscience, University

College London, 17 Queen Square,

London WC1N $3 A R$, UK

e-mail:k.kampe@ucl.ac.uk

$\dagger$ Wellcome Department of Cognitive Neurology,

12 Queen Square, London WC1N 3BG, UK

1. Bruce, V. \& Young, A. Br. J. Psychol. 77, 305-327 (1986).

2. Kleinke, C. L. Psychol. Bull. 100, 78-100 (1986).

3. Emery, N. J. Neurosci. Biobehav. Rev. 24, 581-604 (2000).

4. Schultz, W., Dayan, P. \& Montague, P. R. Science 275,

1593-1599 (1997).

5. Berridge, K. C. Neurosci. Biobehav. Rev. 20, 1-25 (1996)

6. Schultz, W. J. Neurophysiol. 80, 1-27 (1998). 
Quist and Chapela reply - Our original publication $^{1}$ contained two separate conclusions derived from two methodological approaches. First, using PCR, we detected the presence of three distinct transgenic DNA sequences in maize landraces in Oaxaca, Mexico ${ }^{1}$. Second, we attempted to establish the genomic context of transgene insertion using i-PCR. The criticisms raised by Metz and Fütterer and by Kaplinsky et al. relate principally to our second statement.

In contrast with the well-established PCR method, i-PCR is an exploratory method that depends on interpretation and the availability of known sequences in databases such as GenBank. We acknowledge that our critics' assertion of the misidentification of sequences labelled with $a d h 1$ intron 1 and with bronze1 is valid.

The suggestion of mispriming in our i-PCR reaction is also warranted for sequences AF434756 and AF434759 (ref. 1). Significant homology with putative misamplifications is maintained across the length of these fragments, and the CaMV sequence was not recovered. However, this pattern is not found in our other i-PCR sequences. A revealing pattern of discontinuity is found at at least one end of five other sequences, indicating the integration junction between the transgenic DNA and the native host genome. Our critics choose not to recognize this feature in the majority of our i-PCR data. Partial homology with retrotransposon elements in maize is common in primers designed to amplify transposon-like sequences, and is not unique to our primers. Questions concerning the distortion of expected footprints at the DNA-integration junction certainly warrant future work.

The movement of transgenes into new populations and across generations is expected to result in diverse integration patterns $s^{2-7}$. Our findings are compatible with recent studies $^{2-6}$ that characterize transgene/host DNA junctions where rearrangements include interspersion with host or unidentifiable DNA. As altered DNA species should also be an important focus of ecological research, we disagree with our critics who assume that only intact transgenes are worthy of attention in our study.

We agree that PCR-based methods are sensitive and therefore open to artefacts, but strongly disagree that the presence of these artefacts is unavoidable or uncontrollable. The consistent performance of our controls, as reported ${ }^{1}$, discounts beyond reasonable doubt the possibility of false positives in our results. Nevertheless, the high sensitivity of the PCR reaction has incited some critics to request a non-PCRbased method to confirm our main statement. To address these challenges, we

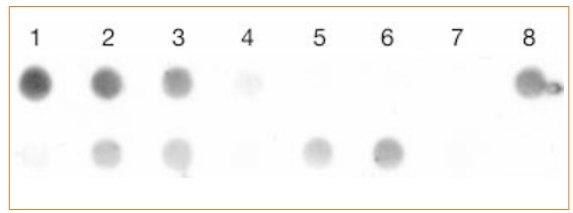

Figure 1 DNA-DNA dot-blot hybridization between maize genomic DNA and a CaMV p-35S probe. Sample numbers coincide with those in ref. 1. Top row: 1, 100\% transgenic; 2, 10\% transgenic; 3, 5\% transgenic; 4, 1\% transgenic, 5, 0.5\% transgenic; 6 , historical maize negative control; 7 , water negative control; 8, Diconsa sample K1. Bottom row: 1, criollo sample B1; 2, criollo sample B2; 3, criollo sample B3; 4, criollo sample A1; 5, criollo sample $A 2 ; 6$, criollo sample $A 3 ; 7$, Peru maize negative control P1; 8, water negative control.

evaluated the same samples from our original publication ${ }^{1}$ using DNA-DNA hybridization. The results of these experiments continue to support our primary statement.

Our analysis of Oaxacan maize is unique for several reasons. First, we wished to document changes that occur within diverse populations of landraces (rather than single varieties or lines), for which no markers, restriction-enzyme digestion maps or linkage analyses have been developed. Second, we could not have predicted which (or how many) specific transgenic constructs (or derivatives) were present in the samples that we analysed. Third, our samples of ground, pooled kernels from individual maize cobs do not represent individual genomes. All of these factors render the application of DNA-hybridization methods difficult. To minimize confusion in interpreting the multiplicity of bands that would have been created by Southern hybridization with our samples, we chose to use dot blotting for our experiments.

We extracted genomic DNA from dry maize kernels ${ }^{1}$. Standards containing varying amounts of transgenic material were prepared by mixing flour from our positive control (Bt1) and our historical negative control $^{1}$. We blotted and immobilized $10-15 \mu \mathrm{g}$ of DNA from each sample onto a nylon membrane using a Bio-Dot apparatus (Bio-Rad). We generated a horseradish peroxidase-labelled DNA probe from the same 220-base-pair fragment of the p-35S CaMV promoter that was amplified from our previously reported ${ }^{1}$ positive control (Bt1). Hybridization conditions were as follows: $56{ }^{\circ} \mathrm{C}, 6 \mathrm{ng} \mathrm{ml}^{-1}$ DNA probe, 1 hour. Washes were as follows: $3 \times 5 \mathrm{~min}$ with $0.1 \times \mathrm{SSC} / 0.1 \% \mathrm{SDS}$ at $56^{\circ} \mathrm{C}$, followed by $3 \times 5 \mathrm{~min}$ with $2 \times \mathrm{SSC}$ at room temperature. Loading homogeneity was confirmed by stripping and rehybridization of the experimental membrane with the 329-base-pair fragment from the maizespecific zein gene ${ }^{1}$. Probe labelling, hybridizations and detection were carried out using a North2South kit (Pierce Endogen), according to the manufacturer's specifications.

DNA from four of our six criollo landrace samples, and from the Diconsa sample, hybridized with our CaMV probe (Fig. 1). By using standardized mixtures of transgenic and non-transgenic maize, dot-blot hybridization suggests a ratio of transgenic to non-transgenic kernels in criollo cobs of the order of 1:100, as we had previously suggested ${ }^{1}$ and as was confirmed by Mexican government studies ${ }^{1}$. This DNA-hybridization study confirms our original detection of transgenic DNA integrated into the genomes of local landraces in Oaxaca.

\section{David Quist, Ignacio H. Chapela}

Department of Environmental Science, Policy and Management, University of California, Berkeley,

California 94720-3110, USA.

e-mail:ichapela@nature.berkeley.edu

1. Quist, D. \& Chapela, I. H. Nature 414, 541-543 (2001).

2. Kohli, A., Gahakwa, D., Vain, P., Laurie, D. A. \& Christou, P. Planta 208, 614 (1999).

3. Kumar, S. \& Fladung, M. Mol. Gen. Genet. 264, 20-28 (2000).

4. Gorbunova, V. \& Levy, A. A. Nucleic Acids Res. 25, 4650-4657 (1997).

5. Windels, P., Taverniers, I., Depicker, A., Van Bockstaele, E. \& De Loose, M. Eur. Food Res. Technol. 213, 107-112 (2001).

6. Pawlowski, W. P. \& Somers, D. A. Proc. Natl Acad. Sci. USA 95, 12106-12110 (1998).

7. Register, J. C. et al. Plant Mol. Biol. 25, 951-961 (1994).

Published online 4 April 2002; DOI 10.1038/nature740

\section{correction}

\section{Reward value of attractiveness and gaze}

K. K. W. Kampe, C. D. Frith, R. J. Dolan, U. Frith

Nature 413, 589 (2001)

Reward-related responses have been registered in animal brains mostly in the ventral half of the striatum, from the nucleus accumbens to the pallidum. Considering the location of the response to attractive faces we describe, the observed activation was large and its spatial extent was not clear from Fig. 2, although we inferred that the ventral stratum was involved. From the plane shown, this activation more accurately extended ventrally into the striatum, specifically into the palladium; the nucleus accumbens proper was not activated. Dorsally, the activation extended into the anterior thalamus (as shown in Fig. 2). Our conclusions that the attractiveness of faces is processed in brain regions involved in evaluating the reward value of stimuli, and that this processing depends on gaze direction, are unaltered. Competing financial interests: declared none.

brief communications is intended to provide a forum for both brief, topical reports of general scientific interest and technical discussion of recently published material of particular interest to non-specialist readers. Priority will be given to contributions that have fewer than 500 words, 10 references and only one figure. Detailed guidelines are available on Nature's website (www.nature.com) or on request from nature@nature.com 